\title{
Bilateral pleural effusions
}

\section{Emmet E. McGrath MB PhD, Chris Barber MD}

Previously published at www.cmaj.ca

See also clinical image by Yang and Liu, page 1883

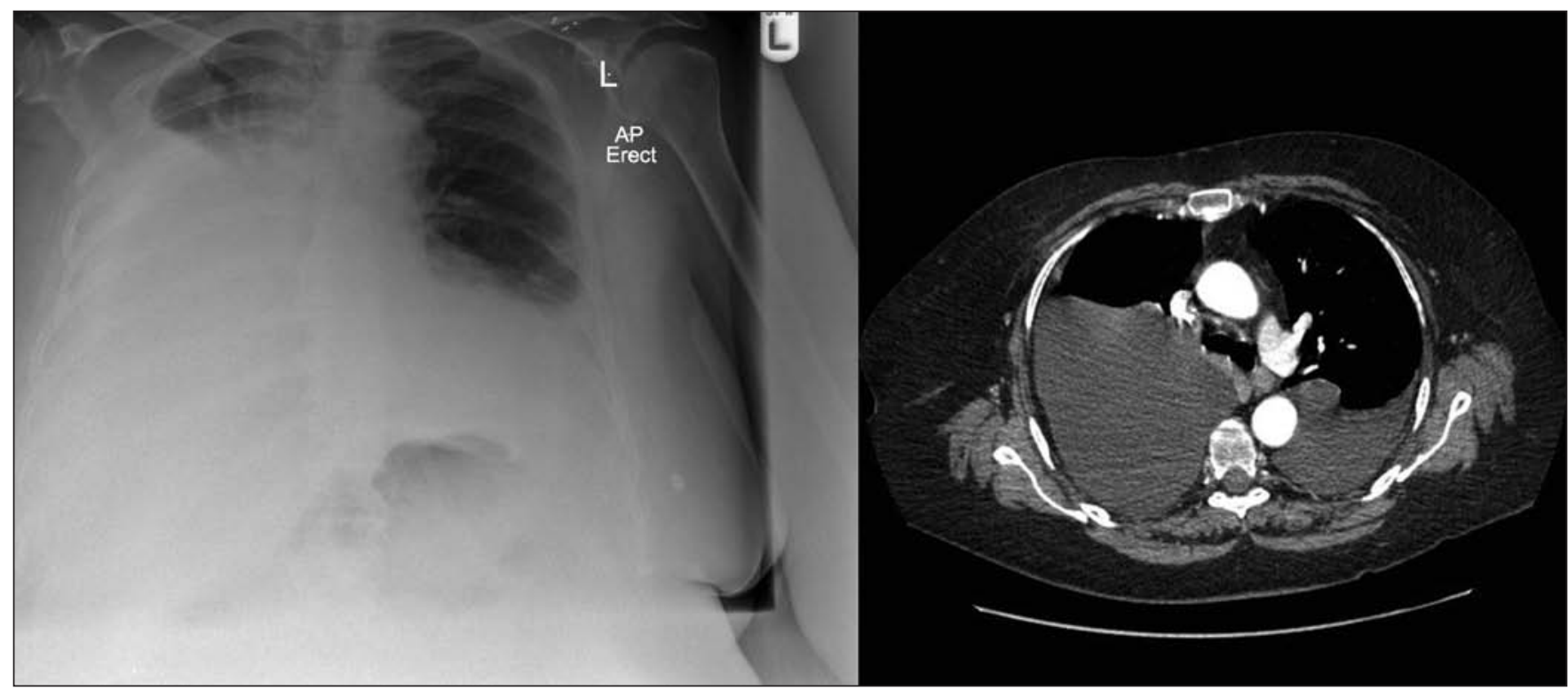

Figure 1: Chest radiograph and contrast-enhanced computed tomography scan of the thorax showing bilateral pleural effusion in a 50 year-old woman with diffuse large B-cell lymphoma.

$\mathrm{A}$ 50-year-old woman presented with a short history of loss of weight and appetite, constipation, rightsided abdominal pain, unsteady gait and headache. Physical examination revealed a soft nontender abdomen with normal bowel sounds. The results of respiratory, cardiovascular and neurologic examinations were normal, as were the findings of a colonoscopy.

A computed tomography (CT) scan of the abdomen showed a large solid mass in the region of the superior mesenteric vessels. A radiograph and CT scan of the thorax were normal. A contrast-enhanced CT scan of the brain showed a large tumour on the left side of the posterior cranial fossa. The results of histological analysis of a biopsy specimen of the brain lesion indicated diffuse large B-cell lymphoma.

Over the very short period of investigation, the patient had dyspnea on minimal exertion without chest pain or hemoptysis. A repeat chest radiograph and CT scan of the thorax showed bilateral pleural effusions (more so on the right side) (Figure 1).

Because the pleural effusions were uneven and there was no history or clinical evidence of cardiac, liver or renal failure, thoracentesis was performed. Milky off-white to yellow fluid was aspirated (Figure 2). It had a protein level of $10 \mathrm{~g} / \mathrm{L}$, a lactate dehydrogenase level of $152 \mathrm{IU} / \mathrm{L}$, a glucose level of $8.1 \mathrm{mmol} / \mathrm{L}$ and a normal $\mathrm{pH}$ level. In serum samples, the total protein level was 48 (normal 60-85) $\mathrm{g} / \mathrm{L}$ and the lactate dehydrogenase level was 858 (normal 105-333) U/L.

\section{What is your diagnosis?}
a. Cardiac failure
b. Pulmonary embolism
c. Malignant pleural disease
d. Complicated parapneumonic effusion
e. Chylothorax

From the Department of Respiratory Medicine, Royal Hallamshire Hospital, Sheffield, UK

CMAJ 2010. DOI:10.1503/cmaj.090920 


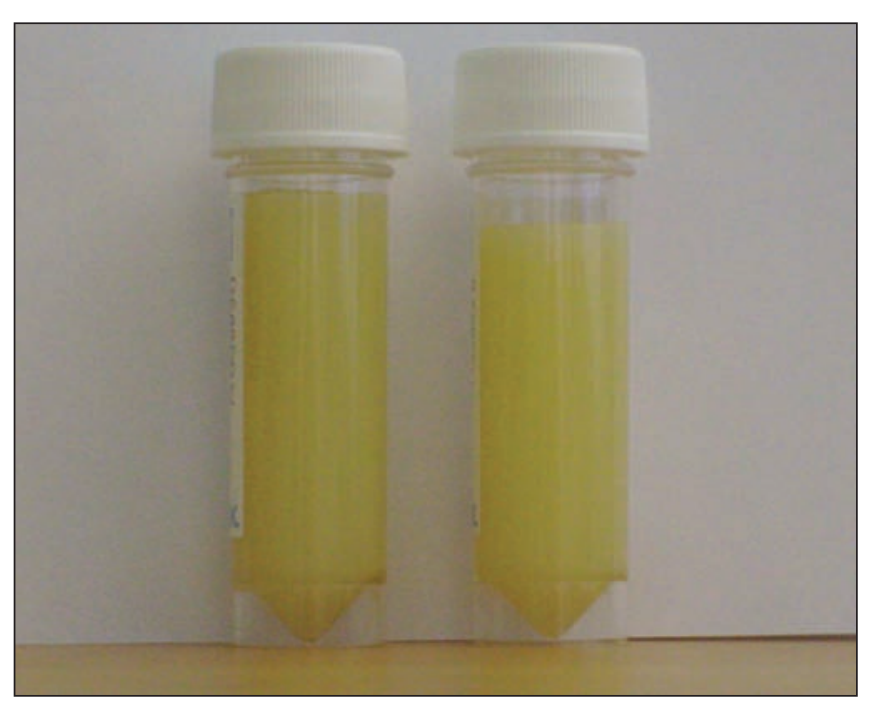

Figure 2: Milky off-white to yellow pleural aspirate.

The answer is (e) chylothorax. Milky white pleural fluid is a classic finding (although appearance may vary) in chylothorax or pseudochylothorax, a condition in which a chronic exudative effusion leads to the accumulation of cholesterol in the pleural fluid. This finding directs the physician to perform specific tests on the fluid to reveal the diagnosis. The distinctive appearance of the fluid is helpful in directing suspicion away from a complicated parapneumonic effusion, which is often associated with yellow turbid pleural fluid.

\section{What is the next most appropriate diagnostic test or procedure?}

a. Echocardiography

b. Cytological analysis of pleural fluid

c. Microbiological staining of pleural fluid

d. Analysis of cholesterol and triglyceride levels in pleural fluid

e. Microbiological culture of pleural fluid

All of the above investigations might be considered appropriate at this point. In our patient, the test that led to the actual diagnosis was (d) analysis of cholesterol and triglyceride levels in pleural fluid, which were $0.3 \mathrm{mmol} / \mathrm{L}$ and $5.2 \mathrm{mmol} / \mathrm{L}$ respectively. Although not required to confirm the diagnosis, echocardiography and routine microbiological and cytological analyses of the fluid were performed to rule out other possible causes (Table 1).

The patient had a chest drain inserted on the right side to allow re-expansion of the lung, which improved her dyspnea. She was transferred to an oncology hospital for further treatment and eventually died because of her malignant disease.

\section{Discussion}

Chylothorax results when chyle leaks in to the pleural cavity as a result of damage or obstruction to the thoracic duct. As lymph vessels from the peritoneal cavity and lower body come together below the diaphragm, they give rise to the

\section{Key points}

- Chylothorax may be unilateral or bilateral depending on the site of leak in the thoracic duct.

- The classic appearance of the pleural fluid in chylothorax is milky white, but it may be serous, yellow or bloody.

- Cholesterol and triglyceride levels in the pleural fluid are used to diagnose chylothorax, with the gold standard lipoprotein analysis used where available, particularly if results are borderline.

- Treatment is usually conservative, with surgery reserved for patients who have large or persistent leaks, have undergone esophagectomy or have become nutritionally compromised.

cisterna chyli, from which the thoracic duct originates. The duct passes with the aorta through the diaphragm and ascends to the subclavian vein (Figure 3). The thoracic duct carries chyle, a combination of lymph and ingested fats, from the intestine.

The most common cause of chylothorax is malignant disease, with lymphoma (in particular, non-Hodgkin's lymphoma) being the most prevalent type encountered. ${ }^{1}$ Trauma, especially as a result of surgery (e.g., esophageal surgery), is the second most common cause of chylothorax.'

Nonmalignant, nontraumatic conditions such as tuberculosis, amyloidosis, lymphangiomyomatosis, filariasis, obstruction of the superior vena cava, retrosternal goitre, hemangiomatosis, yellow nail syndrome and sarcoidosis can also lead to chylothorax. ${ }^{2}$

\section{Is the milky-white colour diagnostic?}

The colour of the pleural fluid can be misleading. The milky off-white to yellow appearance occurs in only about $50 \%$ of patients with chylothorax, with the remainder showing serous, serosanguinous, yellow or bloody fluid. ${ }^{3}$ It can also occur in pseudochylothorax. Laboratory analysis is therefore necessary to confirm the diagnosis.

Biochemical criteria exist for the diagnosis of chylothorax. A triglyceride level greater than $1.24 \mathrm{mmol} / \mathrm{L}$ and a cholesterol level less than $5.18 \mathrm{mmol} / \mathrm{L}$ are diagnostic. ${ }^{3}$ Pleural fluid with a triglyceride level greater than $1.24 \mathrm{mmol}$ has less than a $1 \%$ chance of not being chylous. ${ }^{3}$ Detection of chylomicron

Table 1: Routine and additional analyses performed on pleural fluid ${ }^{4}$

\begin{tabular}{|c|c|}
\hline Routine analysis & Additional analysis \\
\hline $\mathrm{pH}$ & $\begin{array}{l}\text { Amylase (pancreatitis or oesophageal } \\
\text { rupture) }\end{array}$ \\
\hline Glucose & $\begin{array}{l}\text { Cholesterol and triglyceride } \\
\text { (chylothorax) }\end{array}$ \\
\hline LDH & Adenosine deaminase (tuberculosis) \\
\hline Protein & Centrifuge (chylothorax v. empyema) \\
\hline $\begin{array}{l}\text { Microbiological } \\
\text { analysis }\end{array}$ & $\begin{array}{l}\text { Complement (systemic lupus } \\
\text { erythematosus) }\end{array}$ \\
\hline Cytological analysis & Hematocrit (pleural hemorrhage) \\
\hline
\end{tabular}


by lipoprotein analysis is the "gold standard" investigation, but it is not routinely available in many medical centres. It is helpful, however, in borderline cases when the triglyceride level is between 0.56 and $1.24 \mathrm{mmol} / \mathrm{L}$.

\section{What else could it be?}

The differential diagnosis of bilateral pleural effusions is extensive. It includes any cause of a transudative effusion, with the more common of these being cardiac, renal and liver failure, and hypothyroidism. The differential diagnosis also includes certain causes of exudative effusions, such as malignant disease, tuberculosis and pneumonia (Box 1). ${ }^{4}$

Classifying an effusion as a transudate or an exudate requires that the total protein level in the fluid is less than (transudate) or greater than (exudate) $30 \mathrm{~g} / \mathrm{L}$. In patients whose protein level is close to $30 \mathrm{~g} / \mathrm{L}$, the use of Light's criteria is recommended for accurate classification (Box 2).5

In our patient, her history and the radiologic evidence of extensive and rapid accumulation of the pleural fluid pointed to the more common conditions of cardiac, renal or liver failure and was not in keeping with the slow accumulation of fluid usually seen in malignant pleural disease. The echocardiogram did not show any overt signs of cardiac disease or pulmonary embolism. The repeat CT scan of the thorax did not show obstruction of the superior vena cava, pulmonary embolus or malignant pleural deposits. Results of cytologic examination of the pleural fluid were also normal, which in conjunction with the findings from the CT scan, did not point to malignant pleural disease. Serum albumin levels, and renal and liver profiles were normal, which ruled out hypoalbuminemia, and liver or renal disease. Complicated parapneu-

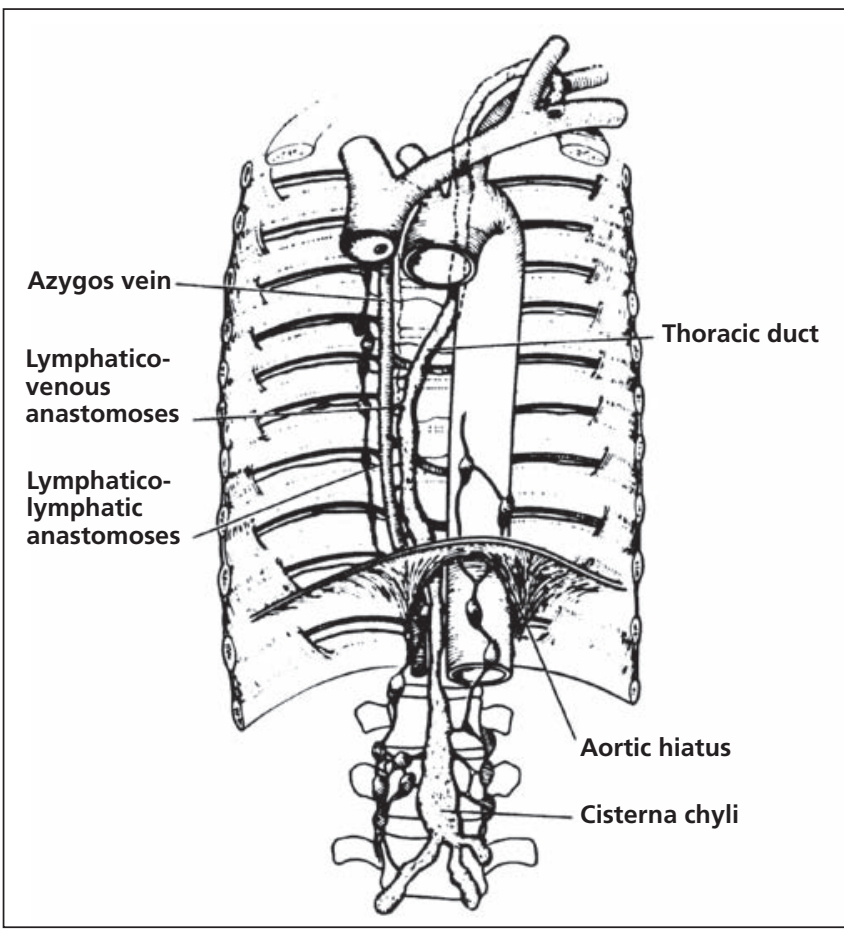

Figure 3: The pathway of the thoracic duct. Reprinted with permission.' Copyright $\odot 1992$ American College of Chest Physicians. monic effusion became part of the differential diagnosis given the colour and consistency of the pleural fluid, even though bilateral complicated parapneumonic effusions are rare. This diagnosis was ruled out because the $\mathrm{pH}$ level in the pleural fluid was normal, the protein level was in the transudative range and results of the microbiologic analysis were normal.

Chylothorax was high on the differential diagnosis because the effusion was milky off-white to yellow, bilateral, and of sudden onset in a patient with lymphoma. Although chylothorax is typically classified as an exudative effusion, transudates have been described in patients with lymphoproliferative disease, following surgery, following exposure to radiation, in idiopathic cases and in patients with concomitant illness such as cardiac or renal failure. ${ }^{6}$

The clinical presentation and past history of our patient did not fit with the other important cause of milky white effusion, pseudochylothorax. The most common conditions associated with pseudochylothorax are tuberculosis, rheumatoid pleurisy or pneumothorax. Pseudochylothorax was ruled out because the high cholesterol and low triglyceride levels in the pleural fluid required for its diagnosis were not found in our patient.

\section{Box 1: Differential diagnosis of bilateral pleural effusion}

Cardiac failure and other causes of transudative effusions (e.g., liver or renal failure, hypothyroidism, hypoalbuminemia, constrictive pericarditis, hypothyroidism)

- Transudate (protein level $<30 \mathrm{~g} / \mathrm{L}$ or negative Light's criteria [Box 2])

- Usually bilateral

- Typically straw-coloured fluid

Malignant pleural disease

- Exudate (protein level $>30 \mathrm{~g} / \mathrm{L}$ or positive Light's criteria [Box 2])

- Usually unilateral

- Slow accumulation of fluid

- Fluid often bloody

Complicated parapneumonic effusion

- Exudate

- Usually unilateral

- Often yellow/green purulent fluid with low pH

- Fluid may have putrid odour

Chylothorax

- Exudate (usually)

- Unilateral or bilateral depending on site of leak

- Fluid is typically milky white, but may be serous, yellow, green or bloody

- Elevated triglyceride and low cholesterol levels

Pseudochylothorax

- Usually unilateral

- Similar appearance to chylothorax

- Low triglyceride and elevated cholesterol levels

Pulmonary embolism

- Transudate

- Unilateral or bilateral (depending on number and site of pulmonary emboli)

- Fluid often bloody

- Often history of chest pain, hemoptysis or painful swelling in legs 


\section{Box 2: Light's criteria for classification of an effusion ${ }^{5}$}

Pleural fluid is an exudate if one or more of the following criteria are met:

- Protein level in pleural fluid divided by level in serum is greater than 0.5 .

- Lactate dehydrogenase level in pleural fluid divided by level in serum is greater than 0.6.

- Lactate dehydrogenase level in pleural fluid is more than two-thirds the upper limit of the normal level in serum.

\section{What treatment options are available?}

Treatment of chylothorax can be conservative or surgical. Conservative treatment is generally considered in patients with minimal chyle leak and few symptoms, because the leak stops spontaneously in up to $50 \%$ of these patients. Conservative treatment involves replacing lost nutrients and reducing the flow of chyle through the thoracic duct, allowing the duct to heal. This is achieved by eliminating dietary fat intake and supplementing nutrition with oral mediumchain triglycerides, or by total parenteral nutrition. ${ }^{3}$ The insertion of a chest drain may also be required to remove the pleural fluid and allow re-expansion of the lung.

Surgery is generally undertaken if there is a persistent leak for more than two weeks, or when chyle leaks at a rate greater than $1.5 \mathrm{~L}$ per day in an adult or greater than $100 \mathrm{~mL} / \mathrm{kg}$ of body weight per day in a child for five days. ${ }^{1}$ Surgery is also usually performed in patients who experience nutritional complications, such as electrolyte and protein depletion. When a large chylothorax occurs rapidly following surgical trauma (e.g., after esophagectomy), surgery may be required. ${ }^{3}$ The site of the leak may be located using lymphangiography or a fat meal mixed with methylene blue dye. ${ }^{3}$ Surgical intervention may involve pleural washout, pleurodesis, pleurectomy or ligation of the thoracic duct. In frail patients unsuitable for major surgery, pleurodesis with talc administered via a chest drain or pleuroperitoneal shunt may be beneficial. ${ }^{3}$ In malignant disease, chemotherapy and radiotherapy may be of benefit. ${ }^{3}$

This article has been peer reviewed.

Competing interests: None declared.

\section{REFERENCES}

1. Valentine VG, Raffin TA. The management of chylothorax. Chest 1992;102:586-91.

2. Hillerdal G. Chylothorax and pseudochylothorax. Eur Respir J 1997;10:1157-62.

3. Nair SK, Petko M, Hayward MP. Aetiology and management of chylothorax in adults. Eur J Cardiothorac Surg 2007;32:362-9.

4. Maskell NA, Butland RJ. BTS guidelines for the investigation of a unilateral pleural effusion in adults. Thorax 2003;58(Suppl 2):ii8-17.

5. Light RW. Clinical practice. Pleural effusion. N Engl J Med. 2002;346:1971-7.

6. Maldonado F, Hawkins FJ, Daniels CE, et al. Pleural fluid characteristics of chylothorax. Mayo Clin Proc 2009;84:129-33.

Correspondence to: Dr. Emmet E. McGrath, Department of Respiratory Medicine, Royal Hallamshire Hospital, Glossop Road, Sheffield S102GF,UK; e.mcgrath@sheffield.ac.uk

\section{ECZEMA FLARES ARE ALWAYS WAITING TO ATTACK.}

